\title{
Spin rate distribution of small asteroids
}

P. Pravec ${ }^{\text {a }}$, A.W. Harris ${ }^{\mathrm{b}}$, D. Vokrouhlický ${ }^{\mathrm{c}}$, B.D. Warner ${ }^{\mathrm{d}}$, P. Kušnirák ${ }^{a}$, K. Hornoch ${ }^{a}$, D.P. Pray ${ }^{\mathrm{e}}$, D. Higgins ${ }^{\mathrm{f}}$, J. Oey ${ }^{\mathrm{g}}$, A. Galád ${ }^{\text {h,a }}, \breve{S}_{\text {. Gajdoš }}{ }^{\mathrm{h}}$, L. Kornoš ${ }^{\mathrm{h}}$, J. Világi ${ }^{\mathrm{h}}$, M. Husárik $^{\mathrm{i}}$, Yu.N. Krugly ${ }^{j}$, V. Shevchenko ${ }^{j}$, V. Chiorny ${ }^{j}$, N. Gaftonyuk ${ }^{k}$, W.R. Cooney, Jr. ${ }^{\ell}$, J. Gross ${ }^{\ell}$, D. Terrell ${ }^{\ell, m}$, R.D. Stephens ${ }^{n}$, R. Dyvig ${ }^{0}$, V. Reddy ${ }^{p}$, J.G. Ries ${ }^{\mathrm{q}}$, F. Colas $^{\mathrm{r}}$, J. Lecacheux ${ }^{\mathrm{s}}$, R. Durkee ${ }^{\mathrm{t}}, \mathrm{G}$. Masi ${ }^{\mathrm{u}, \mathrm{v}}$, R.A. Koff ${ }^{\mathrm{w}}$, R. Goncalves ${ }^{\mathrm{x}}$

${ }^{a}$ Astronomical Institute, Academy of Sciences of the Czech Republic, Fričova 1, CZ-25165 Ondřejov, Czech Republic

${ }^{\mathrm{b}}$ Space Science Institute, 4603 Orange Knoll Ave., La Canada, CA 91011, USA

${ }^{\mathrm{c}}$ Institute of Astronomy, Charles University, Prague, V Holešovičkách 2, CZ-18000 Prague 8, Czech Republic

d Palmer Divide Observatory, 17995 Bakers Farm Rd., Colorado Springs, CO 80908, USA

${ }^{\mathrm{e}}$ Carbuncle Hill Observatory, P.O. Box 946, Coventry, RI 02816, USA

${ }^{\mathrm{f}}$ Hunters Hill Observatory, Ngunnawal, Canberra, Australia

${ }^{\mathrm{g}}$ Leura Observatory, Leura, N.S.W., Australia

${ }^{\mathrm{h}}$ Modra Observatory, Department of Astronomy, Physics of the Earth, and Meteorology, FMFI UK, Bratislava SK-84248, Slovakia

${ }^{i}$ Astronomical Institute of the Slovak Academy of Sciences, SK-05960 Tatranská Lomnica, The Slovak Republic

${ }^{\mathrm{j}}$ Institute of Astronomy of Kharkiv National University, Sumska Str. 35, Kharkiv 61022, Ukraine

${ }^{\mathrm{k}}$ Crimean Astrophysical Observatory, Simeiz, Crimea, Ukraine

${ }^{\ell}$ Sonoita Research Observatory, Sonoita, AZ, USA

${ }^{\mathrm{m}}$ Southwest Research Institute, 1050 Walnut St., Suite 300, Boulder, CO 80302 $U S A$

${ }^{\mathrm{n}}$ Goat Mountain Astronomical Research Station, Yucca Valley, CA

${ }^{\circ}$ Badlands Observatory, Quinn, SD 57775, USA

${ }^{\mathrm{p}}$ Department of Earth System Science and Policy, University of North Dakota, Grand Forks, ND 58202, USA

${ }^{\mathrm{q}}$ McDonald Observatory, USA

${ }^{\mathrm{r}} I M C C E-C N R S$-Observatoire de Paris, 77 avenue Denfert Rochereau, 75014, Paris, France 
${ }^{\mathrm{s}}$ LESIA-Observatoire de Meudon, Place Jules Janssen, 92195 Meudon, France

${ }^{\mathrm{t}}$ Shed of Science Observatory, Minneapolis, MN

${ }^{\mathrm{u}}$ Bellatrix Astronomical Observatory, Via Madonna de Loco 47, I-03023 Ceccano, Italy

${ }^{\mathrm{v}}$ Campo Catino Observatory, Guarcino, I-03016, Italy

${ }^{\mathrm{w}}$ Antelope Hills Observatory, 980 Antelope Drive West, Bennett, CO 80102, USA ${ }^{x}$ Linhaceira, Tomar, Portugal

Version: 2008 May 2, revised. 
Proposed running head: Spin rates of small asteroids

Editorial correspondence to:

Dr. Petr Pravec

Astronomical Institute AS CR

Fričova 1

Ondřejov

CZ-25165

Czech Republic

Phone: 00420-323-620352

Fax: 00420-323-620263

E-mail address: ppravec@asu.cas.cz 


\begin{abstract}
The spin rate distribution of main belt/Mars crossing (MB/MC) asteroids with diameters $3-15 \mathrm{~km}$ is uniform in the range from $f=1$ to $9.5 \mathrm{~d}^{-1}$, and there is an excess of slow rotators with $f<1 \mathrm{~d}^{-1}$. The observed distribution appears to be controlled by the Yarkovsky-O'Keefe-Radzievskii-Paddack (YORP) effect. The magnitude of the excess of slow rotators is related to the residence time of slowed down asteroids in the excess and the rate of spin rate change outside the excess. We estimated a median YORP spin rate change of $\approx 0.022 \mathrm{~d}^{-1} / \mathrm{Myr}$ for asteroids in our sample (i.e., a median time in which the spin rate changes by $1 \mathrm{~d}^{-1}$ is $\approx 45 \mathrm{Myr}$ ), thus the residence time of slowed down asteroids in the excess is $\approx 110 \mathrm{Myr}$. The spin rate distribution of near-Earth asteroids (NEAs) with sizes in the range $0.2-$ $3 \mathrm{~km}$ ( $\sim 5$-times smaller in median diameter than the MB/MC asteroids sample) shows a similar excess of slow rotators, but there is also a concentration of NEAs at fast spin rates with $f=9-10 \mathrm{~d}^{-1}$. The concentration at fast spin rates is correlated with a narrower distribution of spin rates of primaries of binary systems among NEAs; the difference may be due to the apparently more evolved population of binaries among $\mathrm{MB} / \mathrm{MC}$ asteroids.
\end{abstract}

Key words: Asteroids, rotation; Photometry; Near-Earth objects; Satellites of asteroids 


\section{Introduction}

Rotations of asteroids have been set and altered by several processes during their formation and evolution. Large asteroids (with diameter $D>40 \mathrm{~km}$ ) show a Maxwellian distribution of their normalized spin rates, which is consistent with a relaxed distribution due to collisional evolution. Asteroids smaller than $D=40 \mathrm{~km}$ have spin rate distributions different from Maxwellian, with excesses of both slow and fast rotators (Pravec and Harris 2000, Pravec et al. 2002). Until recently, data on rotations of small asteroids have been rather limited; below $D \sim 15 \mathrm{~km}$, there was good and consistent data for near-Earth asteroids only (see the summary in Pravec et al. 2007). Data on spin rates of small main belt asteroids were sparse, as there was no systematic program to obtain them in sufficient number and quality until recently.

In 2004, two dedicated projects of photometric studies of small main belt asteroids were started. Brian Warner began his project of lightcurve observations of Hungaria asteroids. Hungarias are a group of bright (geometric albedos mostly in the range 0.2-0.4) asteroids just outside the orbit of Mars; they are the smallest non-planet crossing asteroids that can be studied with small photometric telescopes. Warner has obtained data on spin rates for more than 80 Hungarias (Warner and Harris 2007). Their preliminary analysis of the sample showed that the Hungaria spin rate distribution is not fundamentally different from the spin rate distribution of near-Earth asteroids. The subset of Brian Warner's sample of Hungarias that satisfies the quality criteria of the BinAstPhotSurvey has been included in the current study. There were 50 such Hungarias.

Since December 2004, we have run the project Photometric Survey for Asynchronous Binary Asteroids (BinAstPhotSurvey, Pravec and Harris 2007, and references therein) that involves a collaboration of a number of asteroid photometrists around the world. Though the main aim of the project has been to detect and describe binary systems among small asteroids, it has also, as a by-product, obtained data on spin rates for nearly 300 main belt and Mars crossing (MB/MC) asteroids with sizes $<15 \mathrm{~km}$. Observations within the BinAstPhotSurvey project have been carried out in a way that largely suppressed selection effects of the photometric technique. In this paper, we present the BinAstPhotSurvey sample of spin rates of small MB/MC asteroids, analyze their distribution, and discuss relationships with theories of evolution of spins of small asteroids and formation of binary systems among them. 


\section{Data set}

In the BinAstPhotSurvey, asteroids with heliocentric semi-major axes $<2.5 \mathrm{AU}$ and absolute magnitudes $H>12$, corresponding to $D<12.5_{-2.3}^{+5.1} \mathrm{~km}$ for geometric albedo $p_{V}=0.18 \pm 0.09$ assumed for asteroids in the inner main belt, ${ }^{1}$ and with favorable observing conditions were selected as observational targets. Lightcurve observations with photometric errors $\leq 0.03$ mag were taken and a sufficient amount of telescope time was allocated for most asteroids so that their periods were uniquely established. The spin rate estimates have been very accurate (relative uncertainties typically on an order of $10^{-4}$ ) for asteroids with periods $<10 \mathrm{~h}$. Noticeable uncertainties or ambiguities occured only in some cases of longer periods where we could not allocate an excessive amount of telescope time. Nonetheless, the presence of lower quality data for some slow rotators did not cause any significant uncertainty in our analyses; a possible mutual contamination between the two slowest bins, $f=0-1 \mathrm{~d}^{-1}$ and $1-2 \mathrm{~d}^{-1}$ in histograms presented below was three objects, but likely only $1-2$, i.e., below statistical uncertainties. The fact that we have paid great attention to obtain good period estimates even for low amplitude asteroids (by giving a large amount of observing time to tough cases) was a key to the success of the project which has provided good period estimates even for asteroids with amplitudes as low as 0.08 mag. For a small fraction (5\%) of targeted asteroids with amplitudes $<0.08 \mathrm{mag}$, we were unable to obtain good period estimates, and they have not been included in the analysis. Since they are so few in number, they could not significantly affect our analyses of spin rate distributions, even in the unlikely case that they might have a non-uniform distribution in $f$.

In the analyses presented below, we have used data for 268 main belt/Mars crossing asteroids with estimated diameters $D=3-15 \mathrm{~km}$. The median diameter of asteroids in the sample is $6.5 \mathrm{~km}$. Only $16 \%$ have $D<4.4 \mathrm{~km}$ and another $16 \%$ have $D>9.8 \mathrm{~km}$, so $68 \%$ of the asteroids in the sample are within a factor of 1.5 of the median diameter. The dataset is available on http://www.asu.cas.cz/ asteroid/binastphotsurv_mbmc_d3_15_071104.txt References for the data in the summary file can be found in the Lightcurve Database compiled by Harris et al., http://www.psi.edu/pds/resource/lc.html and, for data on primaries of binary systems in our sample, in the Binary Asteroid Parameters dataset (see Pravec and Harris 2007).

1 For most asteroids in our sample, direct size estimates were not available. We have estimated their diameters from measured absolute magnitudes $(H)$ and assumed geometric albedos $\left(p_{V}\right)$ using the relation given in Pravec and Harris (2007). Assumed geometric albedos have been taken from Wisniewski et al. (1997): $p_{V}=0.18$ for S, $\mathrm{A}$, and unclassified asteroids, and 0.40 for $\mathrm{V}$ and $\mathrm{E}$ types. For Hungarias without known taxonomic class, we assumed $p_{V}=0.30$ that is about the mean of albedos of $\mathrm{S}$ and $\mathrm{E}$ types that are present among Hungarias in approximately equal fractions. 


\section{Spin rates of small asteroids}

\section{$3.1 \mathrm{MB} / \mathrm{MC}$ asteroids with $\mathrm{D}=3$ to $15 \mathrm{~km}$}

Figures 1 and 2 show a distribution of spin rates of main belt/Mars crossing asteroids with diameters from 3 to $15 \mathrm{~km}$. The distribution is consistent with a uniform distribution between $f=1$ and $9.5 \mathrm{~d}^{-1}$, with an excess of slow rotators at $f<1 \mathrm{~d}^{-1}$. The excess of slow rotators among small asteroids has been found already in previous studies (Pravec and Harris 2000, Pravec et al. 2002) using smaller datasets.

A possible explanation for the uniform distribution of spin rates of small $\mathrm{MB} / \mathrm{MC}$ asteroids between $f=1$ and $9.5 \mathrm{~d}^{-1}$ is provided from the theory of the YORP effect (see, e.g., Capek and Vokrouhlický 2004). The theory predicts that the rate of change of spin frequency $(\dot{f})$ produced by YORP is independent of $f$, as long as it is in a range of frequencies where damping timescales of excited rotation are short in comparison with YORP spin up/spin down timescales. ${ }^{2}$ Any concentration in an original distribution of spin rates is therefore dispersed by the YORP effect, producing a distribution more uniform than the original one. As there is no dependence of $\dot{f}$ on $f$, the evolution of spin rates by the YORP effect does not produce any new concentration in the spin rate distribution. The resulting spin rate distribution is flattened, i.e., it is more uniform than the original distribution.

A simple model showing how a mechanism evolving asteroidal spin rates with $\dot{f}=$ const for each individual asteroid produces a uniform distribution from an original non-uniform one is shown in the appendix. A characteristic timescale $\tau$ of the model corresponds to the YORP doubling/halting time $t_{\mathrm{d}}$ of an asteroid rotating with angular frequency $\omega$ near the middle of the range of spin rates. The doubling/halting time is given by

$$
t_{\mathrm{d}}=\frac{\omega}{|\langle\dot{\omega}\rangle|}=\frac{I_{c} \omega}{\left|\left\langle T_{\omega}\right\rangle\right|}
$$

where $I_{c}$ is the moment of inertia of the asteroid around its principal axis, and $T_{\omega}$ is a component of the torque caused by the YORP effect in the direction of $\omega$ (see Rubincam 2000). Since $t_{\mathrm{d}}$ is, however, by definition related to a specific value of frequency, we prefer to work with $|\langle\dot{f}\rangle| \equiv|\langle\dot{\omega}\rangle| / 2 \pi$ and convert doubling/halting times estimated in other works to $|\langle\dot{f}\rangle|$ using

$$
|\langle\dot{f}\rangle|=\frac{f\left(t_{\mathrm{d}}\right)}{t_{\mathrm{d}}}
$$

2 A basic YORP theory assumes that asteroid is in its basic rotation state around principal axis, i.e., any excitation of rotation produced by the YORP effect is damped down rapidly by inelastic dissipation of energy inside the body. See also comments on coupling between evolution of spin rate and evolution of obliquity in the first paragraph and footnote in Appendix. 
where $f\left(t_{\mathrm{d}}\right)$ is the frequency for which $t_{\mathrm{d}}$ has been estimated.

Čapek and Vokrouhlický (2004) have estimated a median $t_{\mathrm{d}} \approx 12$ Myr for an asteroid with $D=2 \mathrm{~km}$ and bulk density $2.5 \mathrm{~g} / \mathrm{cm}^{3}$ in heliocentric orbit with semi-major axis $a=2.5$ AU, rotating with initial frequency $f\left(t_{\mathrm{d}}\right)=4 \mathrm{~d}^{-1}$ and with obliquity $0 / 180^{\circ}$ (i.e., in asymptotic state), which converts to $|\dot{f}| \approx$ $0.33 \mathrm{~d}^{-1} /$ Myr. Since $|\dot{f}|$ is maximal in the asymptotic state, it represents an upper limit on $|\langle\dot{f}\rangle|$ for asteroids starting with general orientations of their spin vectors. Integrations of YORP evolutions by Vokrouhlický et al. give that $|\langle\dot{f}\rangle|$ for a given asteroid is typically lower by a factor $1.5-2$ than its value of $|\dot{f}|$ in the asymptotic state. So, for the model asteroid of Capek and Vokrouhlický (2004), we get $|\langle\dot{f}\rangle| \approx 0.19 \mathrm{~d}^{-1} / \mathrm{Myr}$. Scaling it to the median $D=6.5 \mathrm{~km}$ and $a=2.26 \mathrm{AU}$ of our MB/MC asteroids sample, we get $|\langle\dot{f}\rangle| \approx 0.022 \mathrm{~d}^{-1} / \mathrm{Myr}$ (i.e., $t_{\mathrm{d}} \approx 180 \mathrm{Myr}$ for asteroid with $f\left(t_{\mathrm{d}}\right)=4 \mathrm{~d}^{-1}$ and a general initial spin axis orientation) as a typical rate of change of frequency for asteroids in our sample. The model presented in Appendix shows that the spin rate distribution is flattened after time $3 \tau$. So, if the asteroids in the MBA/MCs sample are at least $500 \mathrm{Myr}$ old, then the model predicts that the spin rate distribution should be uniform.

According to Bottke et al. (2005), main belt asteroids with $D=3-15 \mathrm{~km}$ have collisional lifetimes of 2 Gyr or longer. Of the 268 asteroids in our sample, 50 and 29 are members of the Hungaria and Phocaea groups, respectively. Asteroids in these two high-inclination asteroidal groups (and moreover with the Hungaria group being decoupled from the main belt) have collisional lifetimes even longer than ordinary main belt asteroids; most of the 79 Hungaria/Phocaea asteroids may be more than 4 Gyr old. Only a few asteroids in the sample that are members of two "recent" families - four belong to the Baptistina family and one belongs to the Massalia family - are younger; the Baptistina and Massalia families are estimated to be 150-200 Myr old (Bottke et al. 2007, Vokrouhlický et al. 2006). So, most asteroids in our sample have probable ages 10-20 times longer than their estimated doubling/halting time $t_{\mathrm{d}}$. It is consistent with the hypothesis that the observed uniform distribution of spin rates between $f=1$ and $9.5 \mathrm{~d}^{-1}$ has been produced by the YORP effect.

An explanation for the excess of slow rotators with $f<1 \mathrm{~d}^{-1}$ is less clear. Generally, a concentration in a certain interval of spin rates is produced when

(1) asteroids originate with spins in the given spin rate interval in higher abundance than outside it, or

(2) rate of a flow of asteroids in the $f$ parameter space is slowed down in the given interval, i.e., asteroids spend longer times in that given interval than in intervals of same width outside of that range. ${ }^{3}$

3 Since we estimate that the collisional age of the small asteroids is many times
longer than the time scale to evolve to slow rotation, the excess of slow rotators
must be in a dynamic equilibrium, i.e., a flow of asteroids into the excess must be
equal to a flow of asteroids out of the excess. If it was not so, e.g., if the flow of 
We speculate that the excess of slow rotators is caused by a generalized YORP effect. The basic YORP theory breaks down at low spin rates where damping timescales of excited rotation are comparable to or longer than YORP evolution timescales for asteroids in the given size range. Therefore, it is possible that for slow rotators, $\dot{f}$ is no longer independent of $f$ as it is for faster rotators. If the YORP effect on slowly rotating asteroids is weakened, they will remain slow rotators for a prolonged time, which would explain the concentration of asteroids at low $f$ 's (scenario 2 above). A first attempt to model the YORP effect on slow rotators by Vokrouhlický et al. (2007) has suggested that the YORP effect itself can trigger tumbling while it spins down an asteroid and then it causes a chaotic evolution of its tumbling rotation. Observationally, studies of non-principal axis (NPA) rotations of asteroids in the size range 1-10 km (Pravec et al. 2005, updated) have shown that at $D \sim 6.5 \mathrm{~km}$, most asteroids with $P>4 \mathrm{~d}$, and some in the range $P=2-4 \mathrm{~d}$ are tumbling. Using these statistics, we estimate that about $1 / 3$ of asteroids in the slow rotators excess are in NPA rotation states. So, a significant part of asteroids in the slow rotators excess may indeed experience a chaotic tumbling rotation evolution by YORP.

An alternative mechanism of excitation of asteroids slowed down by YORP is sub-catastrophic collisions. A characteristic timescale of collisions capable of exciting the rotation of a main belt asteroid with $D=6 \mathrm{~km}$ and $P=4 \mathrm{~d}$ is estimated to be $\approx 70 \mathrm{Myr}$ (adapting formulas given by Farinella et al. 1998). Though the estimated timescale of exciting collisions has a substantial uncertainty (which is dominated by an uncertainty in the estimated number of asteroids with sizes about and above $100 \mathrm{~m}$ ), it is comparable to a median time in which YORP changes spin rates by $1 \mathrm{~d}^{-1}$, that is $\approx 45 \mathrm{Myr}$ (derived as an inversion of $|\langle\dot{f}\rangle| \approx 0.022 \mathrm{~d}^{-1} / \mathrm{Myr}$ given above). So, it is possible that sub-catastrophic collisions contribute to the excitation of slowly rotating asteroids in the excess with $f<1 \mathrm{~d}^{-1}$ with diameters about $6 \mathrm{~km}$ of our MB/MC asteroids sample.

The time of residence $\left(t_{\text {sre }}\right)$ of an asteroid in the slow rotators excess can be estimated by

$$
t_{\text {sre }}=\frac{N_{\text {sre }}}{n_{\text {reg }}|\langle\dot{f}\rangle|},
$$

where $N_{\text {sre }}$ is a number of asteroids in the slow rotators excess, and $n_{\text {reg }}$ and $|\langle\dot{f}\rangle|$, respectively, are a number density and a mean rate of change of rotation frequency of asteroids in the regular spin rate range outside the slow rotators excess. For our sample, we have $N_{\text {sre }}=56, n_{\text {reg }}=23.9 / \mathrm{d}^{-1}$, and $|\langle\dot{f}\rangle| \approx$ $0.022 \mathrm{~d}^{-1} / \mathrm{Myr}$, that gives $t_{\text {sre }} \approx 110$ Myr. We point out that the estimation of the residence time does not depend on an actual amount of contribution of sub-catastrophic collisions to excitation of slow rotators, as long as a typical

asteroids out of the slow rotators excess was zero, we would expect that fully half of all small asteroids (the ones that chanced to have $\dot{f}<0$ ) would accumulate in the slowest spin rate bin. 
magnitude of a spin rate change by exciting impact is less than the width of the slow rotators excess $\left(\sim 1 \mathrm{~d}^{-1}\right)$.

\subsection{Near-Earth asteroids with $D>0.2 \mathrm{~km}$}

A comparison of the data for $3-15 \mathrm{~km}$ sized $\mathrm{MB} / \mathrm{MC}$ asteroids with data for another population of small asteroids, near-Earth asteroids, brings further useful insights. We use the dataset of NEA spin rates from Pravec et al. (2007). The median diameter of NEAs in the sample is $1.3 \mathrm{~km}$, and $85 \%$ of them have $D<3 \mathrm{~km}$. So, the NEA dataset is a sample of smaller asteroids than the sample of small $\mathrm{MB} / \mathrm{MC}$ asteroids given in the previous section; there is very little overlap between the NEA and the MBA/MC samples, and the median $D$ of the NEA sample is 5-times less than the median $D$ of the MBA/MC sample. Interpretations of differences seen between distributions of spin rates of $\mathrm{MB} / \mathrm{MC}$ asteroids and near-Earth asteroids have to take into account their different sizes.

Figure 3 shows a distribution of spin rates of near-Earth asteroids with $D>$ $0.2 \mathrm{~km}$. There are apparent excesses of slow rotators with spin rates $f<1 \mathrm{~d}^{-1}$ and of fast rotators with $f=9-10 \mathrm{~d}^{-1}$ in front of the spin barrier at $f \sim 11 \mathrm{~d}^{-1}$ (see Pravec et al. 2007). The structure near the middle of the range, where there is an apparent concentration at $f \sim 4 \mathrm{~d}^{-1}$ and a drop of the observed density for $f>5 \mathrm{~d}^{-1}$, is at frequencies where data in the NEA spin rates sample may be particularly sensitive to observational selection effects; we will discuss it in the last paragraph of this section.

The excess of slow rotators among NEAs is in the same range of spin rates and it has the same magnitude as for the $\mathrm{MB} / \mathrm{MC}$ asteroids, within the statistical uncertainty. (There are 44 NEAs with $f<1 \mathrm{~d}^{-1}$ and a mean number density in the range $f=1-11 \mathrm{~d}^{-1}$ is $22.8 / \mathrm{d}^{-1}$.) It suggests a common mechanism causing asteroids in both populations (over the size range $0.2-15 \mathrm{~km}$ ) to concentrate at low $f$ 's. It may be the chaotic tumbling rotation evolution by YORP for asteroids in the slow rotators excess (the generalized YORP effect) as discussed for larger MB/MC asteroids in Sect. 3.1, but a possible contribution to excitation of slow rotators from sub-catastrophic impacts needs to be studied.

The excess of fast rotators in front of the spin barrier is not seen among larger MB/MC asteroids. We propose a hypothesis that the fast rotators excess among NEAs is due to primaries of NEA binaries concentrating in the range of the fast rotators excess (see Pravec et al. 2006). In Figs. 2 and 3, we have plotted distributions of binaries among 3-15 km MBA/MCs and NEAs, respectively (the dark colored histograms plotted over the light colored ones which are for all asteroids in the samples). The distribution of spin rates of binary primaries apparently correlates with the distribution of all asteroid spin rates both among NEAs and MBAs/MCs in the range $6-11 \mathrm{~d}^{-1}$. The excess of fast rotating NEAs coincides with the concentration of spin rates of primaries 
of NEA binaries (at periods 2.2-2.8 h, where about 2/3 of NEAs are binary; Pravec et al. 2006), but among MBA/MCs, both the fast rotators excess and the concentration of binary primaries appear dispersed over a broad range (to the degree that the fast rotators excess is no longer visible). If binary systems among both MBAs and NEAs have been formed at the spin barrier, the difference between the distributions - narrow and located in front of the spin barrier for NEAs, and broad, dispersed towards lower rates for MBAs - implies that rotations of primaries of MBA binaries are more evolved from the spin barrier than NEA binaries. Since YORP works slower in larger asteroids, either apparently more evolved MBA binaries are much older than NEA binaries (see also a discussion on lifetimes of binaries in Pravec and Harris 2007), or spins of primaries of binary systems have been dispersed from the spin barrier by another mechanism with a rate greater for larger primaries (i.e., with size dependence opposite to YORP that has a strength inversely proportional to $\left.D^{2}\right)$.

A cause for the third apparent concentration at $f \sim 4 \mathrm{~d}^{-1}$ and the drop of the observed density at $f=5 \mathrm{~d}^{-1}$ is unclear. We consider that it may be largely due to observational biases in the NEA spin rates sample. The mean lightcurve amplitude decreases by a factor greater than 2 with frequencies increasing from $4 \mathrm{~d}^{-1}$ to $7 \mathrm{~d}^{-1}$, with the steepest decrease in the range $5-6 \mathrm{~d}^{-1}$ (see Pravec and Harris 2000, Fig. 6). The apparent drop of the observed density at $f=5 \mathrm{~d}^{-1}$ may be at least in part due to a selection effect against low-amplitude asteroids in the NEA sample. The density at $f \sim 4 \mathrm{~d}^{-1}$ might be also enhanced with respect to lower $f$ 's because the photometric technique as applied in some NEA photometry programs favored detections of relatively short periods about $6 \mathrm{~h}$ against longer ones that generally require a larger amount of observations to be established. The NEA sample is not so homogeneous as the BinAstPhotSurvey sample for MBA/MCs and the observational biases there were not so suppressed as in the BinAstPhotSurvey.

\section{Conclusions}

Spin rates of asteroids smaller than $15 \mathrm{~km}$ appear heavily evolved by the Yarkovsky-O'Keefe-Radzievskii-Paddack effect and processes at the fast spin barrier. Timescales of the YORP evolution are short in comparison with probable ages of small asteroids, so their spin rate distribution appears relaxed and information on initial distribution of spin rates of small asteroids (after their formation in collision events in the main belt, presumably) has been erased.

As large asteroids with $D>40 \mathrm{~km}$ appear collisionally evolved, presumably during their formation period in early Solar System - their normalized spin rates have a Maxwellian distribution - there occurs an interesting question of how the evolution processes affect spin rates of asteroids in the intermediate size range. Scaling the median doubling/halting timescale estimated by Čapek and Vokrouhlický (2004), we get that it is about equal to their ages ( $\sim 4$ Gyr) 
for asteroids with $D \sim 30 \mathrm{~km}$. The range $D=10-40 \mathrm{~km}$ is where the first prominent deviations of spin rate distribution from Maxwellian appear, going down from larger sizes. There occur first several "outlying" slow rotators, indicating an onset of the slow rotators excess at sizes of a few tens $\mathrm{km}$. The geometric mean spin rate $(\langle f\rangle)$ generally increases (with possible minor wavy variations) in the range with diameter going down from 40 to $10 \mathrm{~km}$ (see Fig. 2 in Pravec and Harris 2000, also Fig. 2 in Pravec et al. 2002). The observed increase of $\langle f\rangle$ with decreasing diameter in the 10-40 $\mathrm{km}$ size range may be due to the increasing rate of the YORP evolution in the range. A levelling of the geometric mean spin rate at $\langle f\rangle=4-5 \mathrm{~d}^{-1}$ in the range $D=1-15 \mathrm{~km}$ that is apparent in the plots mentioned above appears to occur there where the spin distribution is heavily relaxed and asteroid spin rates dispersed over the whole possible range of frequencies (from nearly 0 to $\sim 11 \mathrm{~d}^{-1}$ ) so that there is little further dependence of $\langle f\rangle$ on $D$ in the $1-15 \mathrm{~km}$ size range.

\section{Acknowledgements}

The work at Ondřejov was supported by the Grant Agency of the Czech Republic, Grant 205/05/0604. The work at Space Science Institute (A.W.H.) was supported by grant NAG5-13244 from the NASA Planetary GeologyGeophysics Program. D. Vokrouhlický has been supported by the Research Program MSM0021620860 of the Czech Ministry of Education. Funding for observations at the Palmer Divide Observatory (B.D.W.) has been provided by NASA grant NNG06GI32G, by National Science Foundation grant AST0607505, and by a Gene Shoemaker NEO grant from the Planetary Society. Funding for observations at the Carbuncle Hill Observatory (D. Pray) and at the Hunters Hill Observatory (D. Higgins) has been provided by Gene Shoemaker NEO Grants from the Planetary Society. The work at Modra Observatory was supported by the Slovak Grant Agency for Science VEGA, Grants 1/3074/06 and 1/3067/06. The work at Skalnaté Pleso Observatory (Astronomical Institute SAS, Tatranská Lomnica) was supported by the Slovak Grant Agency for Sciences VEGA, Grant 2/7009/27. We thank to M. Pikler and G. Cervák for observational assistance. The work at McDonald Observatory was supported by NASA's NEO Observation Program Grant NNG04G124G. The work at Pic du Midi Observatory has been supported by CNRS - Programme de Planétologie. We thank to P. Brown, S. Nudds, and Z. Krzeminski for their contribution with observations from Elginfield Observatory, University of Western Ontario. 


\section{APPENDIX}

\section{A Simple model for the YORP-dominated rotation rate dynamics}

Assume a simple model for time evolution of rotation frequency $f$ with constant rate $C$ of its change. Thus, we have

$$
\frac{\mathrm{d} f}{\mathrm{~d} t}=\frac{f_{\mathrm{med}}}{\tau}=C
$$

where $f_{\text {med }}$ is median value of $f$ and $\tau$ is some characteristic timescale. YORP dominated evolution of regular rotation states (SAM rotation mode) has been found to support such a simplified model (e.g., Čapek and Vokrouhlický 2004). ${ }^{4}$ The rotation rate $f$ is defined in some finite interval of values between 0 , for a non-rotating body, and $f_{\mathrm{m}}$, the maximum value corresponding to rotational fission. Solution of Eq. (A.1) would have been trivial if it were not for the boundary conditions at 0 and $f_{\mathrm{m}}$. This is because a simple YORP theory of bodies in the SAM mode breaks down (i) for slow rotation, with possible onset of tumbling and/or other contributing effects such as impacts, and (ii) near the rotation fission limit where the asteroid can change shape due to landsliding or even shed mass followed by satellite formation or escape of its fragments (e.g., Scheeres 2007). Neither of these two regimes is well-understood and we are forced to use a rough approximation of things happening in the two limiting regimes in our model.

The rate $C$ in Eq. (A.1) might be either positive or negative with about the same likelihood (Čapek and Vokrouhlický 2004). This means YORP can either increase or decrease the rotation rate. Moreover, the value of $C$ depends on the degree of irregularity of the asteroid shape, such that it can be very small or zero for smooth-enough surfaces or those endowed with particular symmetries (e.g., triaxial ellipsoids). It is maximized for highly irregular shapes. In what follows we shall assume $C$ values have a Maxwellian distribution with the maximum probability density at some characteristic value $f_{\text {med }} / \tau$ (see Čapek and Vokrouhlický 2004). We also use a formalism in which the sign of $C$ is included into $f$, such that $C$ is always positive, but $f$ is formally defined to have values in an extended interval $\left(-f_{\mathrm{m}}, f_{\mathrm{m}}\right)$. A real, measured physical quantity is $|f|$, and evolution on the negative $f$ branch is that of YORP-deceleration and

4 Note that in a detailed YORP evolution model, a rotation rate evolution would be coupled with evolution of obliquity (e.g., Rubincam 2000; Vokrouhlický and Čapek 2002; Čapek and Vokrouhlický 2004). However, as Čapek and Vokrouhlický (2004) have shown, a typical YORP evolution ultimately drives obliquity into particular asymptotic states. As a result, Eq. (A.1) should be understood as description of the evolution after reaching the asymptotic states. Including the obliquity evolution would mean to add some initial transition phase into the model. We neglect it in the zero-level approximation; we also consider that it may be partially absorbed into the timescale $\tau$. 
evolution on the positive $f$ branch is that of YORP-acceleration. The points $f=0$ and $f=f_{\mathrm{m}}$ are boundaries where specific conditions should determine an exact evolution of the rotation rate $f$ (note $f=-f_{\mathrm{m}}$ cannot be reached by the flow defined in Eq. (A.1)). While a realistic modeling of what happens at both boundaries is beyond the scope of this paper, we adopt the following crude approximations:

- $f=0$. When an evolving object reaches this boundary, we choose a new value of $C$ from its probability distribution, and let the body evolve toward the positive branch of the $f$ axis.

- $f=f_{\mathrm{m}}$. Rotation rate $f$ of an object reaching $f_{\mathrm{m}}$ is set to $f=-f_{\mathrm{m}}$ and its motion continues with a new, randomly chosen $C$ rate along the negative branch of the $f$ axis (it means that it "bounced" at the spin barrier $f_{\mathrm{m}}$ ).

With the identification $f_{\mathrm{m}}$ and $-f_{\mathrm{m}}$ the evolution is effectively that of a flow on a circle. Changing the rate of change $C$ at 0 and $\pm f_{\mathrm{m}}$ produces a shear that tends to smear any initial structures in the rotation rate distribution. A few comments on the above choices are in order.

Letting the solution go from near $f=0$ to positive $f$ values means the solution must re-emerge to nominal rotation rates by acceleration of its rotation frequency. The exact process of how this happens is unknown. It may be driven by sub-catastrophic collisions that can chip off parts of the target and re-shape it so that the direction of the YORP evolution is reversed from previous deceleration to acceleration of $f$. A similar evolution may be, however, driven by YORP itself. Vokrouhlický et al. (2007) have shown that during the chaotic tumbling rotation phase the YORP effect may eventually flip the spin axis in the body frame and orient the body such that it starts gaining rotation energy. When internal dissipation then brings the rotation into a SAM mode, the reverted spin axis in the body frame may cause YORP spin up again. In both cases it seems appropriate to randomly choose a new rate of change $C$ at the very slow rotation mode.

The physical situation near the fission limit is also unknown. For instance, our simple circular flip from $f_{\mathrm{m}}$ to $-f_{\mathrm{m}}$ may describe a situation where a body that has reached the fast spin barrier is re-shaped by shedding small pieces from its surface until YORP starts decelerating its rotation. This model neglects situations such as when the body re-shapes into a near-spherical shape and then stalls near, or evolves slowly from, the spin barrier for a prolonged time. Such a situation appears to occur for primary components of binary systems among small asteroids (see Sect. 3 and references given there).

For the starting $f$ distribution in our simulations, we used a Maxwellian distribution with peak at $f=4$. Since an initial state is quickly forgotten in the simulated evolution, the particular choice of initial distribution does not critically affect the result of the simulations.

Figure 4, left panel, shows the result of our simulation. To minimize statistical fluctuations, we used $10^{6}$ trial evolutions to determine the plotted distribu- 
tions. If we used a lower number of trial cases, for instance 268 objects as is the number of asteroids in the observed MBA/MCs sample, we would obtain qualitatively the same result, but with statistical fluctuations in the calculated distribution due to the limited number of points. A fundamental result is that relaxation toward uniform distribution is achieved at $t$ between $2 \tau$ and $3 \tau$ and after that the distribution shows no more noticeable evolution. The real data show such uniform distribution over most of the spin rate interval, but there is observed the excess at slow rates (Fig. 2). We have speculated above that it has to do with the actual mechanism by which an asteroid reemerges from the very slow rotation state. In our toy model, it means that a delay must be introduced between entering the slow rotators bin and reemerging from it. We heuristically model this process by decreasing a value of $C$ in Eq. (A.1) to another, effective value $C_{1}$ when the object reaches the $|f|<1$ range. Choosing $C_{1}=C / 2$ we get results shown on the right panel of Fig. 4. While the relaxation timescale remains same, the resulting evolved $f$-distribution resembles the one observed for small main belt asteroids.

\section{References}

Bottke, W.F., Durda, D.D., Nesvorný, D., Jedicke, R., Morbidelli, A., Vokrouhlický, D., Levison, H.F., 2005. Linking the collisional history of the main asteroid belt to its dynamical excitation and depletion. Icarus 179, 63-94.

Bottke, W.F., Vokrouhlický, D., Nesvorný, D., 2007. An asteroid breakup 160 Myr ago as the probable source of the K/T impactor. Nature 449, 48-53.

Čapek, D., Vokrouhlický, D., 2004. The YORP effect with finite thermal conductivity. Icarus 172, 526-536.

Farinella, P., Vokrouhlický, D., Hartmann, W.K., 1998. Meteorite delivery via Yarkovsky orbital drift. Icarus 132, 378-387.

Pravec, P., Harris, A.W., 2000. Fast and slow rotation of asteroids. Icarus 148, $12-20$.

Pravec, P., Harris, A.W., Michałowski, T., 2002. Asteroid rotations. In: Bottke Jr., W.F., Cellino, A., Paolicchi, P., Binzel, R.P. (Eds.), Asteroids III. University of Arizona Press, Tucson, pp. 113-122.

Pravec, P., and 19 colleagues, 2005. Tumbling asteroids. Icarus 173, 108-131.

Pravec, P., and 56 colleagues, 2006. Photometric survey of binary near-Earth asteroids. Icarus 181, 63-93.

Pravec, P., Harris, A.W., 2007. Binary asteroid population. 1. Angular momentum content. Icarus 190, 250-259.

Pravec, P., Harris, A.W., Warner, B.D., 2007. NEA rotations and binaries. In Milani, A., Valsecchi, G.B., Vokrouhlický, D. (Eds.), Proc. IAU Symp. 236. 
Cambridge University Press, Cambridge, pp. 167-176.

Rubincam, D.P., 2000. Radiative spin-up and spin-down of small asteroids. Icarus 148, 2-11.

Scheeres, D.J., 2007. Rotational fission of contact binary asteroids. Icarus 189, 370-385.

Vokrouhlický, D., Čapek, D., 2002. YORP-induced long-term evolution of the spin state of small asteroids and meteoroids: Rubincam's approximation. Icarus 159, 449-467.

Vokrouhlický, D., Brož, M., Bottke, W.F., Nesvorný, D., Morbidelli, A., 2006. Yarkovsky/YORP chronology of asteroid families. Icarus 182, 118-142.

Vokrouhlický, D., Breiter, S., Nesvorný, D., Bottke, W.F., 2007. Generalized YORP evolution: Onset of tumbling and new asymptotic states. Icarus 191, 636-650.

Warner, B.D., Harris, A.W., 2007. Lightcurve Studies of Small Asteroids. Bull. Amer. Astron. Soc. 39, 432.

Wisniewski, W.Z., Michalowski, T.M., Harris, A.W., McMillan, R.S., 1997. Photometric observations of 125 asteroids. Icarus 126, 395-449. 


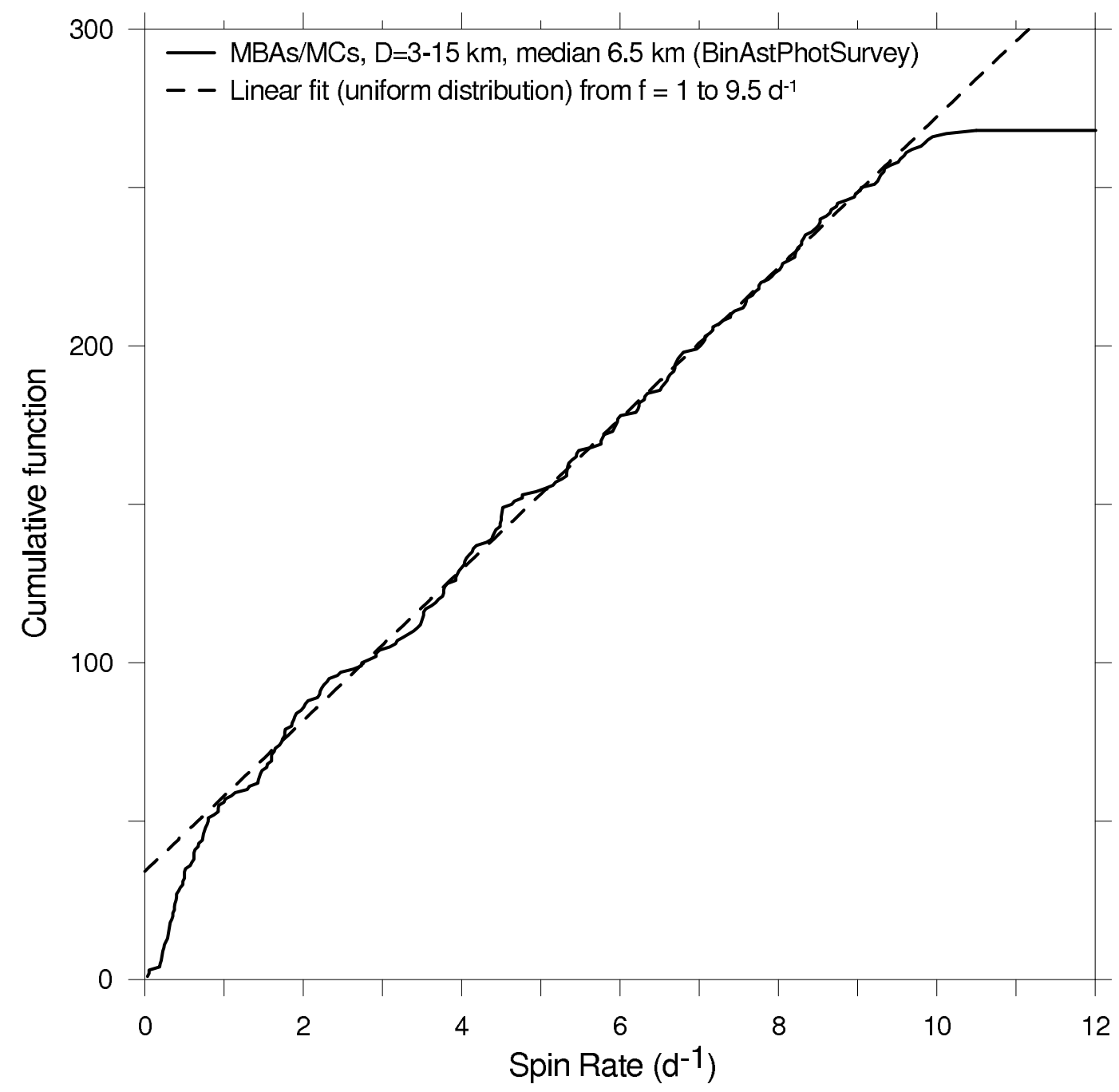

Fig. 1. Cumulative distribution of spin rates of main belt/Mars crossing asteroids with $D=3$ to $15 \mathrm{~km}$. A histogram of the data is shown in Fig. 2. The spin rate distribution is uniform from $f=1$ to $9.5 \mathrm{~d}^{-1}$, and there is an excess of slow rotators at $f<1 \mathrm{~d}^{-1}$ (the steep slope of the cumulative distribution in the interval $0-1 \mathrm{~d}^{-1}$; see also Fig. 2). 


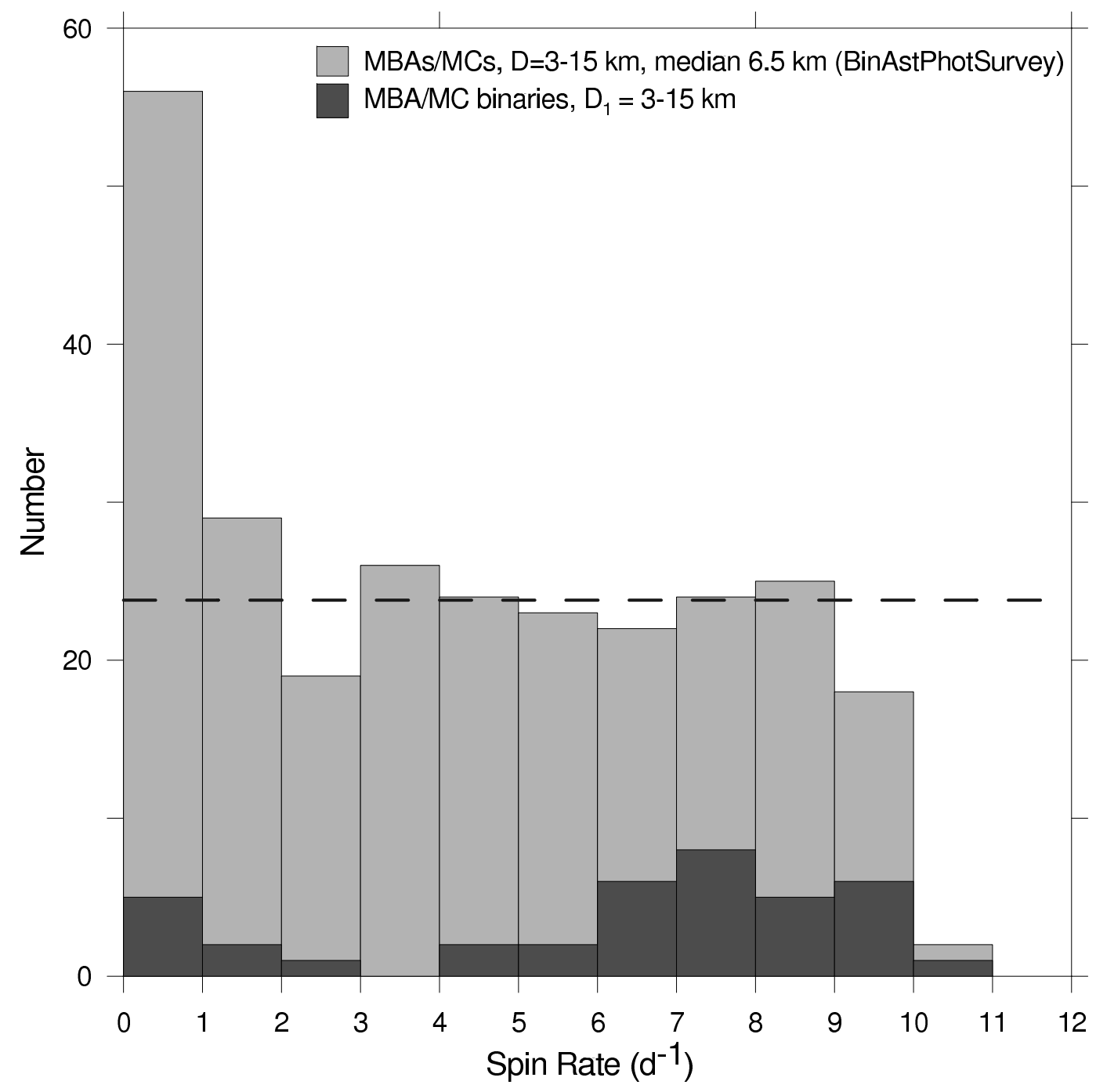

Fig. 2. Histogram of spin rates of main belt/Mars crossing asteroids with $D=3$ to $15 \mathrm{~km}$ (same data as in Fig. 1; see comments in its caption). Lower histogram (dark colored bins, plotted over the grey bins histogram) shows a distribution of spin rates of primaries of all known $\mathrm{MB} / \mathrm{MC}$ binaries with primary diameters $D_{1}=3$ to $15 \mathrm{~km}$. The dashed line indicates a mean number density of $23.9 / \mathrm{d}^{-1}$ in the range $f=1$ to $9 \mathrm{~d}^{-1}$ in the given sample. 


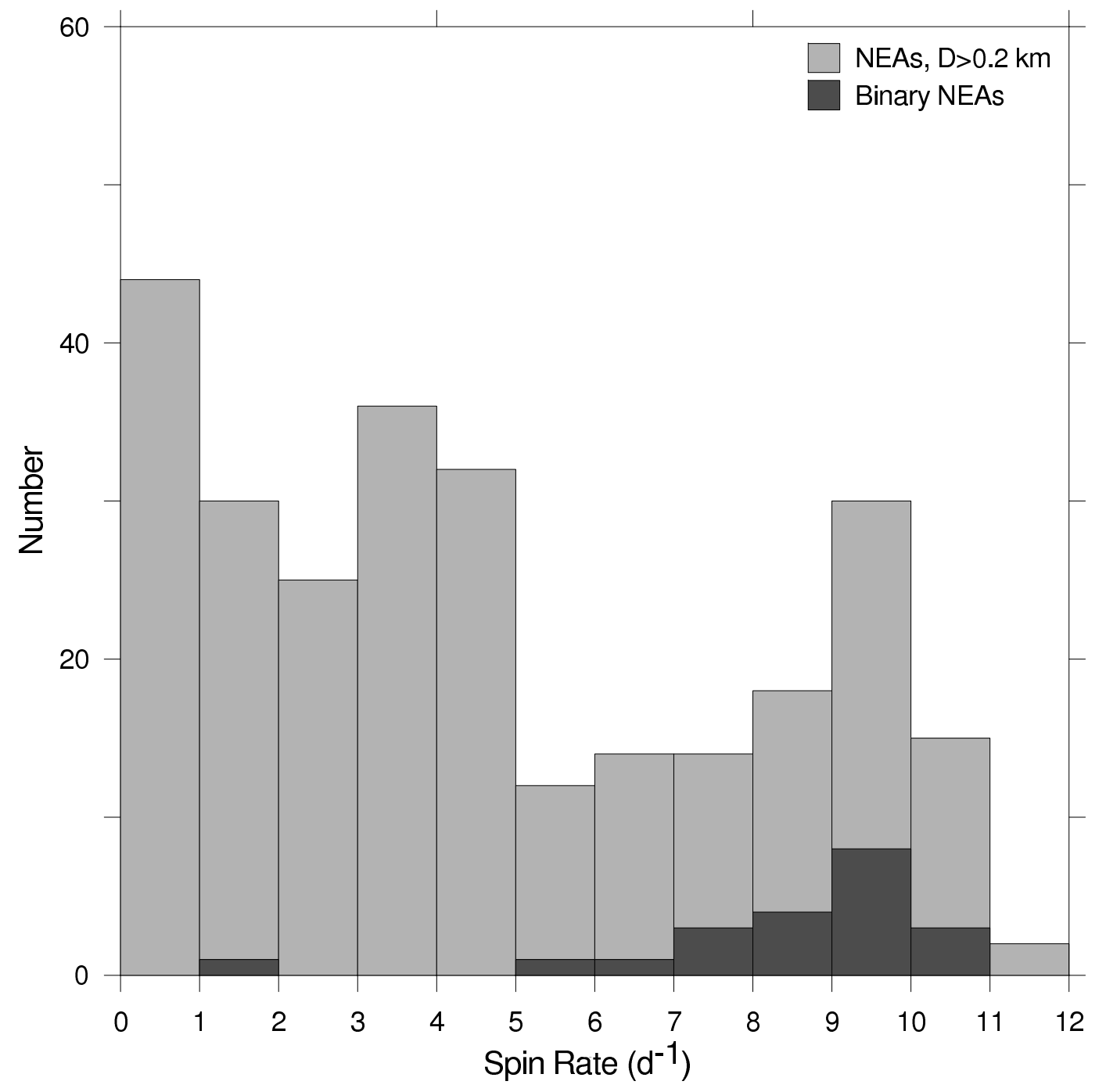

Fig. 3. Histogram of spin rates of near-Earth asteroids, and primaries of NEA binaries, with $D>0.2 \mathrm{~km}$ (update of Fig. 3 in Pravec et al. 2007). The excesses of slow rotators with spin rates $f<1 \mathrm{~d}^{-1}$ and of fast rotators with $f=9-10 \mathrm{~d}^{-1}$ (pile up in front of the spin barrier at $f \sim 11 \mathrm{~d}^{-1}$ ) are apparent. The latter coincides with concentration of spin rates of primaries of NEA binaries. Note that the plotted binary data are observed, they have not been corrected for selection effects. After debiassing, heights of the bins for binaries would be higher by a factor of about 2.5 ; about $66 \%$ of NEAs with periods $2.2-2.8 \mathrm{~h}\left(f \sim 8.6-10.9 \mathrm{~d}^{-1}\right)$ are actually binary (Pravec et al. 2006). Note: One known super-fast rotator, $2001 \mathrm{OE}_{84}$ with $f=49.33 \mathrm{~d}^{-1}$ is off scale of the shown histogram. 

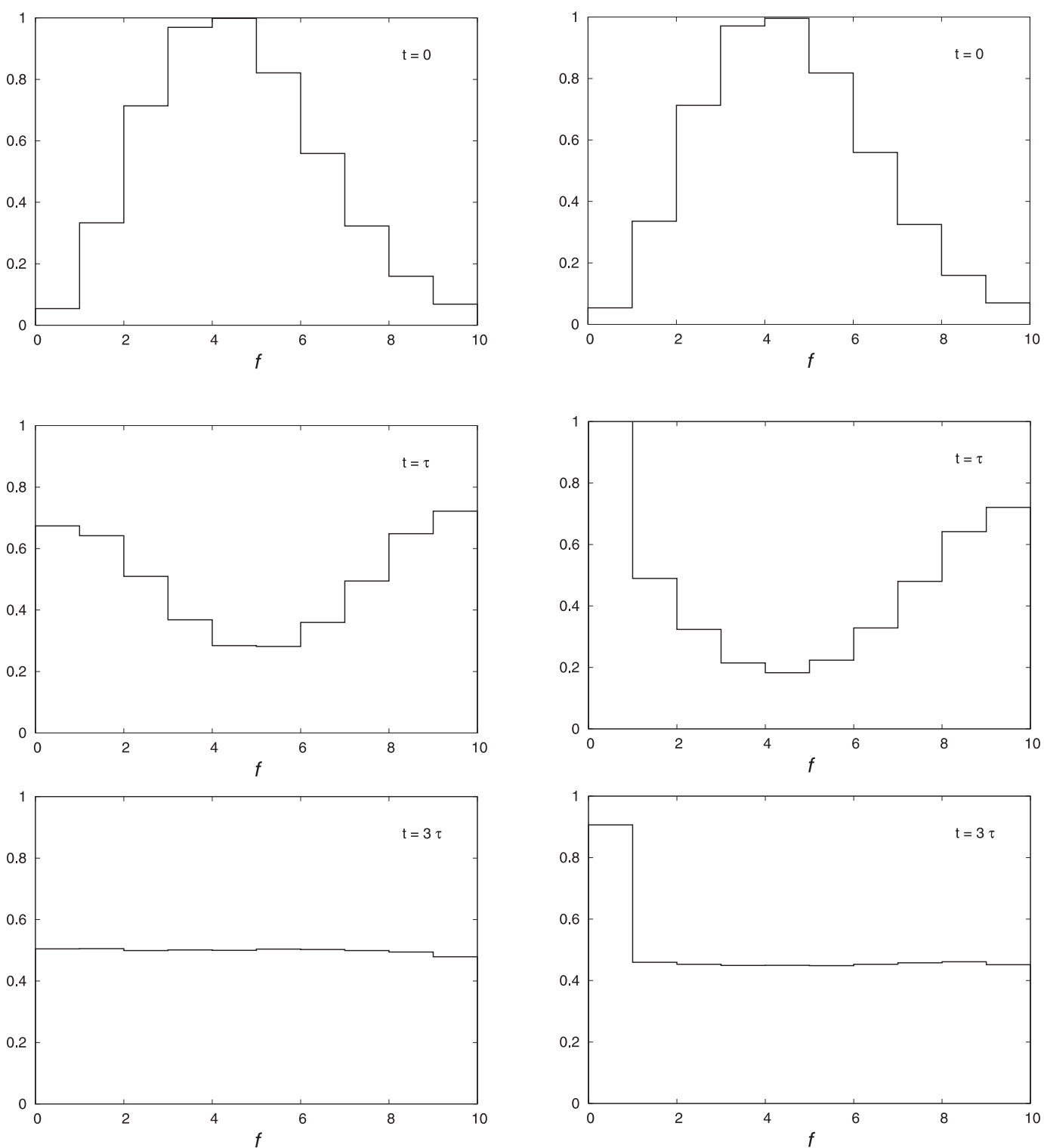

Fig. 4. Simulated spin rate distribution from our simple model at three time instants: (i) at $t=0$, assumed initial distribution (Maxwellian; top panels), (ii) distribution at $t=\tau$ (middle panels), and (iii) distribution at $t=3 \tau$ (bottom panels). Maximum of the distribution has been arbitrarily normalized to unity. Left column: the first model with $C$ constant for individual asteroid as it moves over the whole $f$ range. Right column: the modified model with $C$ in the first bin $f=0-1$ set to half of its value outside the slow rotators bin. 